\title{
Exact quantum dynamics of XXZ central spin problems
}

\author{
Wen-Bin He, ${ }^{1,2}$ Stefano Chesi, ${ }^{3,4, *}$ Hai-Qing Lin, ${ }^{3,4}$ and Xi-Wen Guan ${ }^{1,5,6, \dagger}$ \\ ${ }^{1}$ State Key Laboratory of Magnetic Resonance and Atomic and Molecular Physics, Wuhan Institute of Physics and Mathematics, \\ Chinese Academy of Sciences, Wuhan 430071, China \\ ${ }^{2}$ University of Chinese Academy of Sciences, Beijing 100049, China \\ ${ }^{3}$ Beijing Computational Science Research Center, Beijing 100193, China \\ ${ }^{4}$ Department of Physics, Beijing Normal University, Beijing 100875, China \\ ${ }^{5}$ Center for Cold Atom Physics, Chinese Academy of Sciences, Wuhan 430071, China \\ ${ }^{6}$ Department of Theoretical Physics, Research School of Physics and Engineering, Australian National University, \\ Canberra ACT 0200, Australia
}

(Received 11 October 2018; revised manuscript received 2 May 2019; published 20 May 2019)

\begin{abstract}
We obtain analytically close forms of benchmark quantum dynamics of the collapse and revival (CR), the reduced density matrix, Von Neumann entropy, and fidelity for the XXZ central spin problem. These quantities characterize the quantum decoherence and entanglement of the system with any number of bath spins, and for a short to infinitely long time evolution. For the homogeneous central spin problem, the effective magnetic field $B$, coupling constant $A$, and longitudinal interaction $\Delta$ significantly influence the time scales of the quantum dynamics of the central spin and the bath, providing a tunable resource for quantum metrology. Under the resonance condition $B=\Delta=A$, the location of the $m$ th revival peak in time reaches a simple relation $t_{r} \simeq \frac{\pi N}{A} m$ for a large $N$. For $\Delta=0, N \rightarrow \infty$, and a small polarization in the initial spin coherent state, our analytical result for the CR recovers the known expression found in the Jaynes-Cummings model, thus building up an exact dynamical connection between the central spin problems and the light-matter interacting systems in quantum nonlinear optics. In addition, the CR dynamics is robust to a moderate inhomogeneity of the coupling amplitudes while disappearing at strong inhomogeneity.
\end{abstract}

DOI: 10.1103/PhysRevB.99.174308

Quantum dynamics of many-body systems has been a longstanding challenge in physics [1-5]. It is always a formidable task for physicists due to the difficulty of analytically deriving many-body eigenfunctions and the exponentially growing complexity of numerics [6-8].

Over the past decade, important progress has been made in a variety of fields, such as atomic qubits coupled to a cavity [9-12], central spin problems [7,13-20], resonant superconductor qubits [21-25], and long-range interacting spin chains of Rydberg atoms [26,27]. This has greatly improved our understanding of quantum dynamics and entanglement of many-body systems.

In this context, exact Bethe ansatz solvable models have been particularly fruitful to the study of quantum dynamics of this kind, e.g., integrability-based central spin problems [28-39], atom-field interacting systems in quantum nonlinear optics $[1,40,41]$, thermalization and quantum dynamics [42-45], quantum hydrodynamics [46,47], etc.

However, the problem of the size of the Hilbert space increasing exponentially with the particle number still prohibits full analytical access to the quantum dynamics at the many-body level. Therefore, it is extremely rare to find exact quantum dynamics of integrable models.

\footnotetext{
*stefano.chesi@csrc.ac.cn

${ }^{\dagger}$ xwe105@wipm.ac.cn
}

Here we circumvent the complexity of the usual Bethe ansatz [48] and develop analytical approaches to the homogeneous central spin problems, obtaining a full characterization of their quantum dynamics through simple closed-form expressions. We further substantiate the relevance of the analytical results through exact numerical simulations, showing that the main predicted features, such as the occurrence of collapse and revival (CR) dynamics, are robust to inhomogeneity.

\section{QUANTUM COLLAPSE AND REVIVAL}

One class of integrable systems with long-range interactions, called Richardson-Gaudin models [49-51], has found interesting applications in various physical problems [14,34,35,52]. The XXZ central spin problem, i.e., a central spin coupled to $N$ bath spins, is described by the Hamiltonian

$$
H=B \mathbf{s}_{0}^{z}+2 \sum_{j=1}^{N}\left[A_{j}\left(\mathbf{s}_{0}^{x} \mathbf{s}_{j}^{x}+\mathbf{s}_{0}^{y} \mathbf{s}_{j}^{y}\right)+\Delta_{j} \mathbf{s}_{0}^{z} \mathbf{s}_{j}^{z}\right],
$$

where $B$ is an effective external magnetic field for the central spin [53], $N$ is the number of spins in the bath, $A_{j}$ is the transverse coupling amplitude, and $\Delta_{j}$ is the longitudinal interaction. The model (1) is integrable if $\Delta_{j}$ and $A_{j}$ are related through $\Delta_{j}^{2}-A_{j}^{2}=$ const; see [32,38,39].

Although this type of model, e.g., (1), was known as an exactly solvable model long ago [50], the binomial sets of Bethe ansatz roots $C_{N+1}^{M}$ impose a big numerical challenge in calculating the quantum dynamics of this model $[6,7,36,37]$. 
Here $M$ is the number of total down spins in the system. The importance of Hamiltonian (1) is in its promising applications to realistic problems in quantum metrology, based on nitrogen vacancy (NV) centers [54], highly symmetric molecules with $N$ nuclear spins coupled to the nuclear spin of a central atom [5,55], etc.

The general central spin problem with nonuniform couplings, for example $A_{j}=A \exp [-\alpha(j-1) / N]$, where $\alpha$ is the inhomogeneity parameter, is integrable but its dynamics is still challenging to analyze. We first analytically solve the dynamical evolution for the homogeneous case, namely $A_{j}=$ $A, \Delta_{j}=\Delta$, and later we analyze the effect of inhomogeneity for the CR. The homogeneous central spin problem (see, e.g., Refs. $[14,35,56])$ enables one to derive exact expressions of the quantum dynamics since bath spins can map onto a large spin operator $\mathbf{J}=\sum_{j=1}^{N} \mathbf{s}_{j}$. Thus Eq. (1) can be rewritten as

$$
H=B \mathbf{s}_{0}^{z}+A\left(\mathbf{s}_{0}^{+} \mathbf{J}^{-}+\mathbf{s}_{0}^{-} \mathbf{J}^{+}\right)+2 \Delta \mathbf{s}_{0}^{z} \mathbf{J}^{z} .
$$

Below we analytically derive the CR dynamics, the reduced density matrix, Von Neumann entropy, and fidelity, providing an important benchmark quantum dynamics of this class of models.

The phenomenon of quantum CR has long been studied in quantum nonlinear optics [1]. However, there still lacks a comprehensive understanding of such a phenomenon in interacting spin systems [14,34-37,57]. To overcome the exponentially increasing scales in solving the Bethe ansatz equations of the Gaudin magnet (1), here we directly calculate the wave function under a unitary time evolution of the Hamiltonian (2), i.e., $|\psi(t)\rangle=e^{-i H t}\left|\Phi_{0}\right\rangle$. In the initial state $\left|\Phi_{0}\right\rangle=|\uparrow\rangle_{0} \otimes\left|\Phi_{\text {bath }}\right\rangle \quad$ with $\left|\Phi_{\text {bath }}\right\rangle=\otimes_{j=1}^{N}\left[\sin (\theta / 2)|\uparrow\rangle_{j}+\right.$ $\cos (\theta / 2)|\downarrow\rangle_{j}$ ] (see, e.g., Refs. [14,56]). The spin coherent state can be written in terms of the Dicke states as $\left|\Phi_{\text {bath }}\right\rangle=\sum_{n=0}^{N} \sqrt{C_{N}^{n}}[\sin (\theta / 2)]^{n}[\cos (\theta / 2)]^{N-n}|n\rangle$ with $|n\rangle=$ $\left|\frac{N}{2}, n-\frac{N}{2}\right\rangle$.

The Dicke state is the eigenstate of the operators $\mathbf{J}^{2}$ and $\mathbf{J}^{z}$. For example, $\mathbf{J}^{z}|n\rangle=\left(-\frac{N}{2}+n\right)|n\rangle, \mathbf{J}^{-}|n\rangle=\sqrt{b_{n}}|n-1\rangle$, and $\mathbf{J}^{+}|n\rangle=\sqrt{b_{n+1}}|n+1\rangle$ with $b_{n}=n(N-n+1)$.

We develop a recurrence method to determine the wave function and the dynamical property of the system. After a lengthy calculation, we may obtain an explicit form of the wave function at arbitrary times, i.e.,

$$
\begin{aligned}
|\psi(t)\rangle= & \sum_{n=0}^{N} \sqrt{C_{N}^{n}}[\sin (\theta / 2)]^{n}[\cos (\theta / 2)]^{N-n} \\
& \times\left[P_{\downarrow}^{n}(t)|\downarrow\rangle_{0}|n+1\rangle+P_{\uparrow}^{n}(t)|\uparrow\rangle_{0}|n\rangle\right],
\end{aligned}
$$

where the parameters are defined as follows: $P_{\uparrow}^{n}=-i\left(\Delta_{n+1} / \Omega_{n+1}\right) \sin \left(\frac{\Omega_{n+1} t}{2}\right)+\cos \left(\frac{\Omega_{n+1} t}{2}\right) \quad$ and $\quad P_{\downarrow}^{n}=-$ $i 2\left(\sqrt{b_{n+1}} A / \Omega_{n+1}\right) \sin \left(\frac{\Omega_{n+1} t}{2}\right)$ with $\Delta_{n}=B+(2 n-1-N) \Delta$ and $\Omega_{n}^{2}=\Delta_{n}^{2}+4 b_{n} A^{2}$; see the SM [58] for a detailed derivation.

We observe that the Rabi oscillation frequency $\Omega_{n}$ has an essential dependence on the coupling parameters $A, \Delta$, and the effective magnetic field $B$. The flip-flop interaction, i.e., the second term of Eq. (2), leads to the state change between $|\downarrow\rangle_{0}|n+1\rangle$ and $|\uparrow\rangle_{0}|n\rangle$.

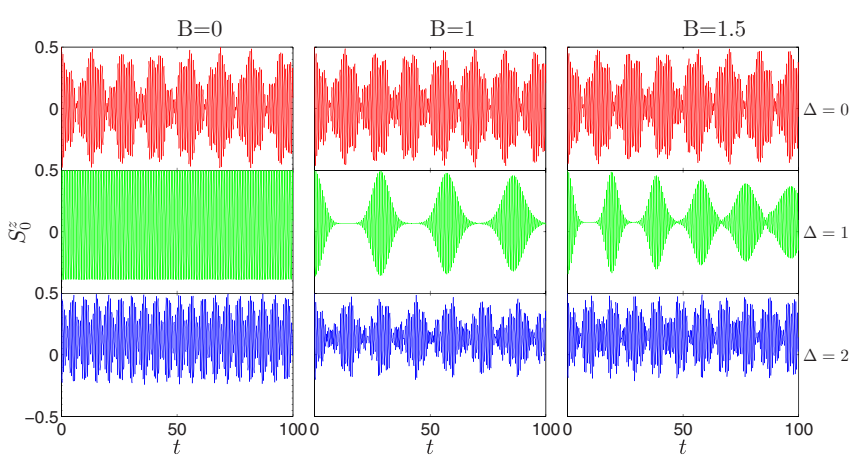

FIG. 1. The central spin polarization evolves in time under different values of the magnetic field $B$ and longitudinal interaction $\Delta$. In contrast to a two-level atom coupled to a cavity [1], here we demonstrate that at resonance $\Delta=A=B=1$, the quantum CR can be observed even for a small system size $N=8$ [for $N=4,6$; see the Supplemental Material (SM) [58]]. Such a small number of bath spins are experimentally accessible, for example by superconducting circuits $[23,24]$.

Using the closed form of the wave function (3), the time evolution of the central spin polarization $S_{0}^{z}(t)=$ $\left\langle\psi(t)\left|\mathbf{s}_{0}^{Z}\right| \psi(t)\right\rangle$ can be calculated in a straightforward way. By a lengthy algebra, we obtain an explicit form of the quantum $\mathrm{CR}$ of the homogeneous central spin problem,

$$
\begin{aligned}
S_{0}^{z}(t)= & \frac{1}{2} \sum_{n=0}^{N} C_{N}^{n}\left[\sin ^{2}(\theta / 2)\right]^{n}\left[\cos ^{2}(\theta / 2)\right]^{N-n} \\
& \times\left[\frac{\Delta_{n+1}^{2}}{\left(\Omega_{n+1}\right)^{2}}+\frac{4 b_{n+1} A^{2}}{\left(\Omega_{n+1}\right)^{2}} \cos \left(\Omega_{n+1} t\right)\right] .
\end{aligned}
$$

This compact form of the quantum CR describes a very rich quantum dynamics of the many-body problems (2); see Fig. 1. The effective magnetic field $B$, the coupling constant $A$, the longitudinal interaction $\Delta$, and the number of bath spins $N$ all play an important role in controlling the features of the quantum CR dynamics. The Rabi oscillation frequency $\Omega_{n}=$ $\sqrt{\Delta_{n}^{2}+4 b_{n} A^{2}}$ depends not only on the Dicke state $n$ but also on the coupling constant $A$ and the longitudinal interaction $\Delta$. For a large $\Delta$ the collapses disappear, whereas the revival period becomes shorter. An almost perfect $\mathrm{CR}$ occurs at the resonance $\Delta=A=B$. The interaction effect, driven by $\Delta$, strongly influences the frequency of the oscillation, the amplitudes of the revivals, and the fidelity of the central spin. It is particularly interesting to observe that a finite $\Delta$ facilitates the quantum revivals even for a very small number of bath spins. This can help with experimental control of quantum dynamic transfers in these kinds of systems; see the SM [58] for a detailed discussion.

Under such a resonance condition $B=\Delta=A$, the oscillation frequency becomes $\Omega_{n}=A \sqrt{4 n+N^{2}}$. Thus the revival peak times $t_{r}$ satisfy a simple relation

$$
\left(\Omega_{n+1}-\Omega_{n}\right) t_{r}=2 \pi m \quad(m=1,2,3, \ldots),
$$

i.e., the neighboring oscillation terms differ by an integer times $2 \pi$. For a large bath size, i.e., $N \gg 1$, we get the location of the $m$ th revival peak in time $t_{r} \simeq \frac{\pi N}{A} m$, which is confirmed in Fig. 2. It is linearly proportional to the bath spin 


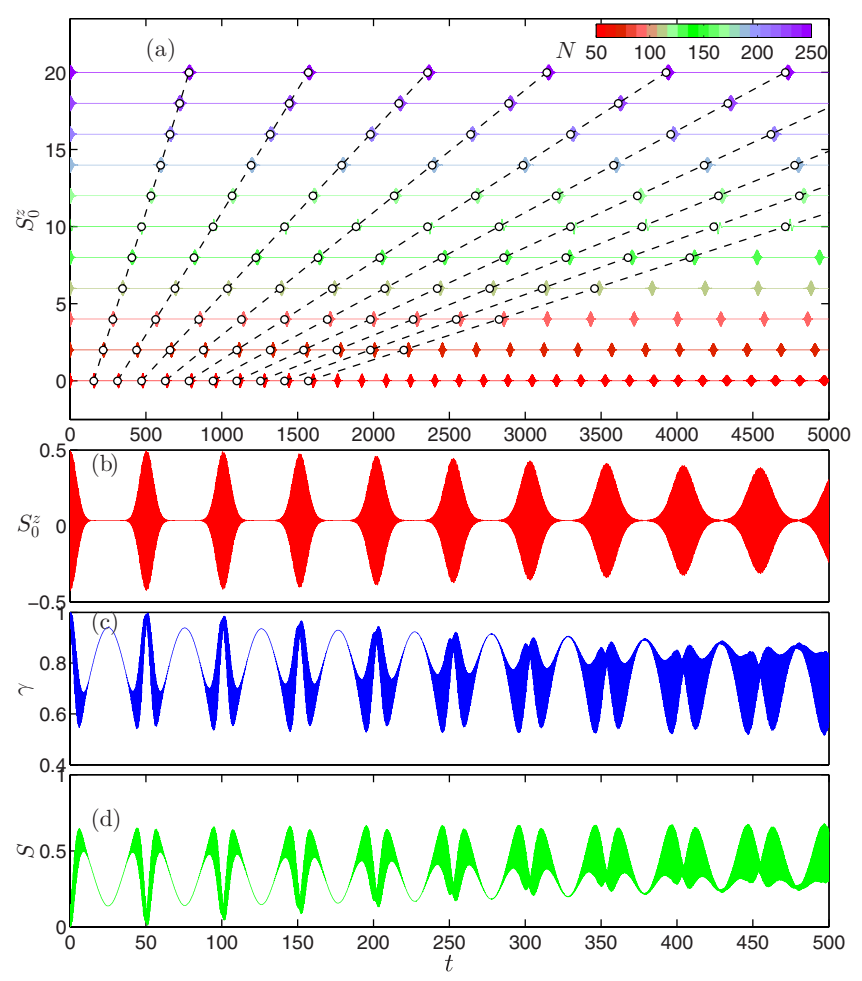

FIG. 2. (a) Time evolution of the central spin polarization for different bath spin sizes $N \in$ [50-250], with particle number step $\delta N=20$. All the curves are shifted upward by 1 for $N>50$. We use the circles and dashed lines to mark the revival peaks. The times of the revival peaks depend linearly on the bath size $N$; see the main text. Time evolution of the central spin polarization for a smaller value of $N=15$ (b), together with the corresponding quantum purity (c) and Von Neumann entropy (d). Here $B=\Delta=A=1$.

number $N$. In Fig. 4, we will discuss how the initial revival dynamics remains almost unaffected by a relatively large inhomogeneity, $\alpha \sim 1$. This $\mathrm{CR}$ dynamics is distinct from the revivals observed in the spin-echo signal of central spin systems, when the evolution time is a multiple of the nuclear Larmor periods [59-61] (thus that revival time is independent of $N)$.

\section{DECOHERENCE AND ENTANGLEMENT}

To characterize the nature of entanglement between the bath and the central spin, we further calculate the reduced density matrix of the central spin by tracing out the degrees of freedom of the bath spins $\{|n\rangle\langle n|\}$,

$$
\rho_{\mathrm{cs}}=\operatorname{Tr}_{\{|n\rangle\langle n|\}}[|\psi(t)\rangle\langle\psi(t)|]=\left(\begin{array}{lc}
A(t) & B(t) \\
B(t)^{*} & 1-A(t)
\end{array}\right),
$$

where the matrix elements read

$$
\begin{aligned}
A(t)= & \sum_{n=0}^{N} C_{N}^{n}\left[\sin ^{2}(\theta / 2)\right]^{n}\left[\cos ^{2}(\theta / 2)\right]^{N-n}\left|P_{\uparrow}^{n}\right|^{2}, \\
B(t)= & \sum_{n=0}^{N} \sqrt{C_{N}^{n+1} C_{N}^{n}}\left[\sin ^{2}(\theta / 2)\right]^{n+\frac{1}{2}}\left[\cos ^{2}(\theta / 2)\right]^{N-n-\frac{1}{2}} \\
& \times P_{\uparrow}^{n+1}\left(P_{\downarrow}^{n}\right)^{*} .
\end{aligned}
$$
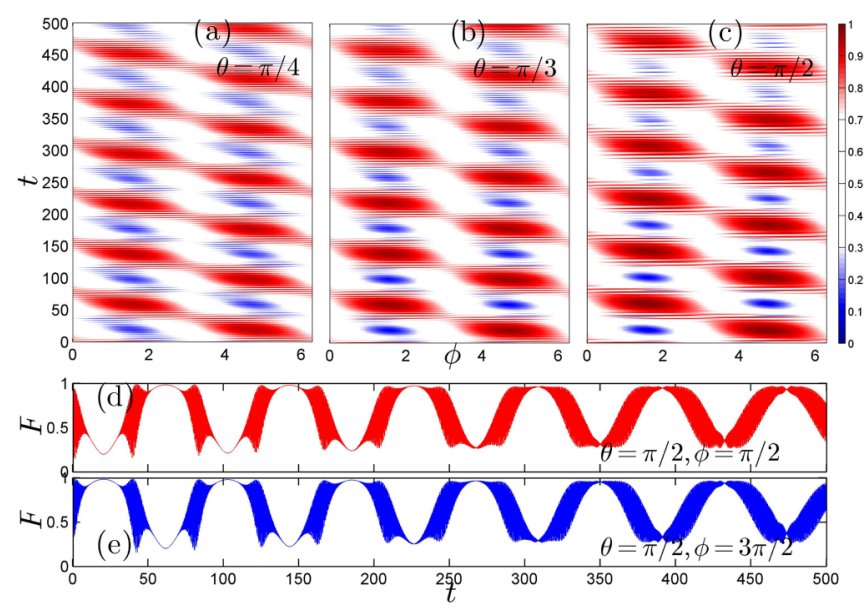

FIG. 3. Contour plots of fidelity vs the phase $\phi$ for different values of $\theta$. (a)-(c) Time evolution of the fidelity of the reduced density matrix of the central spin $\rho_{\mathrm{cs}}$ against the state $|\phi\rangle=\frac{1}{\sqrt{2}}\left[|\uparrow\rangle_{0}+\right.$ $\left.e^{-i \phi}|\downarrow\rangle_{0}\right]$. Panels (d) and (e) show time evolution of the fidelity for the phase angles $\phi=\pi / 2$ and $\phi=3 \pi / 2$. In all panels, $B=\Delta=$ $A=1$.

The purity and Von Neumann entropy, which characterize the entanglement between the central spin and the bath spins, are given explicitly via the relations $\gamma \equiv \operatorname{Tr}\left[\rho_{\mathrm{cs}}^{2}\right]$ and $S\left(\rho_{\mathrm{cs}}\right) \equiv$ $-\operatorname{Tr}\left[\rho_{\mathrm{cs}} \ln \rho_{\mathrm{cs}}\right]$. They are displayed in Figs. 2(c) and 2(d), showing an important decoherence effect: while the central spin entropy (purity) is initially small (large) at the CR points, it gradually increases (decreases) with time. As a consequence, the CR gradually vanish in the long-time limit.

We further look at the fidelity of $\rho_{\mathrm{cs}}$ with respect to the state $|\phi\rangle=\frac{1}{\sqrt{2}}\left(|\uparrow\rangle_{0}+e^{-i \phi}|\downarrow\rangle_{0}\right)$. By definition, the fidelity is given by $F\left(\rho_{\mathrm{cs}}, \rho_{\phi}\right)=\sqrt{\left\langle\phi\left|\rho_{\mathrm{cs}}\right| \phi\right\rangle}$ for the pure state $\rho_{\phi}$, leading to

$$
F=\sqrt{\left[1+B e^{-i \phi}+B^{*} e^{i \phi}\right] / 2} .
$$

In Fig. 3, we contour plot $F$ in the phase-time plane $(\phi, t)$. It is interesting to observe that fidelity oscillations with high contrast occur for the two special values of the phase $\phi=\frac{\pi}{2}$ or $\phi=\frac{3 \pi}{2}$. As seen from Figs. 3(a)-3(c), the optimal choices of $\phi$ are independent of $\theta$ [58]. The fidelity peaks occur around the middle points of the collapse regions and are over $92 \%$ in Figs. 3(d) and 3(e), where $\theta=\pi / 2$. Instead, moving away from $\theta=\pi / 2$ causes a reduction of the maximum fidelity. The slow decay of the oscillations means that the central spin is able to decouple periodically from the bath. A longer decoherence time may facilitate dynamical control of entangled states in NV center devices [54].

\section{STATISTICAL NATURE OF THE GENERALIZED JAYNES-CUMMINGS MODEL}

We now give an exact mapping between the homogeneous XXZ central spin problems and the atom-field interaction model in quantum nonlinear optics. From the Holstein-Primakoff transformation $\mathbf{J}^{+}=\sqrt{N} \mathbf{a}^{\dagger} \sqrt{1-\mathbf{a}^{\dagger} \mathbf{a} / N}$, $\mathbf{J}^{-}=\sqrt{N} \sqrt{1-\mathbf{a}^{\dagger} \mathbf{a} / N} \mathbf{a}$, and $\mathbf{J}^{z}=-\frac{N}{2}+\mathbf{a}^{\dagger} \mathbf{a}$, where $\mathbf{a}\left(\mathbf{a}^{\dagger}\right)$ is a bosonic annihilation (creation) operator, we may build a deep connection between the central spin problems and the 
matter-light interaction systems [1]. In the large- $N$ limit, the Hamiltonian Eq. (2) becomes (up to a constant)

$$
H=B^{\prime} \mathbf{s}_{0}^{z}+\sqrt{N} A\left[\mathbf{s}_{0}^{+} \mathbf{a}+\mathbf{s}_{0}^{-} \mathbf{a}^{\dagger}\right]+2 \Delta \mathbf{s}_{0}^{z} \mathbf{a}^{\dagger} \mathbf{a}+h \mathbf{a}^{\dagger} \mathbf{a},
$$

where the effective magnetic field is $B^{\prime}=B+h$, and $h$ is related to the light frequency [62], whereas $\sqrt{N} A$ is related to the coupling constant between the atom and bosonic mode $[1,40,63]$. This model (8) can be regarded as a generalized Jaynes-Cummings (JC) model, in which the atomic transition frequency also depends on the number of photons. For $\Delta=0$, the central spin problem Hamiltonian (2) exactly reduces to the JC model.

To see a dynamical connection between the two systems, let us define the inversion $W_{\mathrm{cs}}(t)$ of the central spin following the JC model. The inversion is immediately found from Eq. (4), since $W_{\mathrm{cs}}(t)=\left\langle\psi(t)\left|\sigma_{z}\right| \psi(t)\right\rangle=$ $2 S_{0}^{z}$. Using the Poisson limit theorem when $N \rightarrow \infty$ and $p \rightarrow 0$, we have $C_{N}^{n} p^{n}(1-p)^{N-n} \simeq e^{-\lambda} \frac{\lambda^{n}}{n !}$, where $\lambda=N p$. Then, taking the limit $\theta \rightarrow 0$ for the initial angle, we find $C_{N}^{n}\left[\sin ^{2}(\theta / 2)\right]^{n}\left[\cos ^{2}(\theta / 2)\right]^{N-n} \simeq e^{-\zeta^{2}} \frac{\left(\zeta^{2}\right)^{n}}{n !}$, where $\zeta^{2}=$ $N \sin ^{2}(\theta / 2)$. As a consequence, the inversion is given by

$$
\begin{aligned}
W_{\mathrm{cs}}(t)= & \sum_{n=0}^{\infty} e^{-\zeta^{2}} \frac{\left(\zeta^{2}\right)^{n}}{n !} \\
& \times\left[\frac{\Delta_{n+1}^{2}}{\left(\Omega_{n+1}\right)^{2}}+\frac{4(n+1) N A^{2}}{\left(\Omega_{n+1}\right)^{2}} \cos \left(\Omega_{n+1} t\right)\right] .
\end{aligned}
$$

Here the parameters read $\Delta_{n+1}=B-N \Delta$ and $\Omega_{n+1}=$ $\sqrt{\Delta_{n+1}^{2}+4(n+1) N A^{2}}$. This is nothing but an exact result of quantum CR of the generalized JC model (8). Moreover, taking the limit $\Delta \rightarrow 0$, the expression Eq. (9) recovers Eq. (6.2.21) of Ref. [1] for the JC model; see also Fig. S10 in the SM [58]. The exact correspondence between the special case of the XXZ central spin problems (2) and the JC model reads

\begin{tabular}{ccc} 
Central spin model & & JC model \\
\hline$B$ & $\rightarrow$ & $\Delta_{\mathrm{JC}}$ \\
$\sqrt{N} A$ & $\rightarrow$ & $g$ \\
$\zeta^{2}$ & $\rightarrow$ & $\langle n\rangle$
\end{tabular}

where the parameters of the Jaynes-Cummings model are, respectively, the detuning, the coupling between the photon and atom, and the average photon number. This correspondence presents a deep relation between the two types of models, i.e., a large number of bath spins with a particular choice of the spin coherent state $(\theta \rightarrow 0)$ can be regarded as a single-occupied-multilevel fermionic field that naturally reduces to a bosonic field, revealing a statistical nature of the Holstein-Primakoff transformation.

\section{INHOMOGENEOUS CENTRAL SPIN PROBLEM}

To assess the effect of inhomogeneity on the quantum dynamics discussed so far, we diagonalize Eq. (1) with different values of the inhomogeneity parameter $\alpha$. The wave function and central spin polarization for the inhomogeneous model, at arbitrary $M$ and time, are obtained in the SM [58]. We observe that a strong inhomogeneity of the coupling amplitudes leads

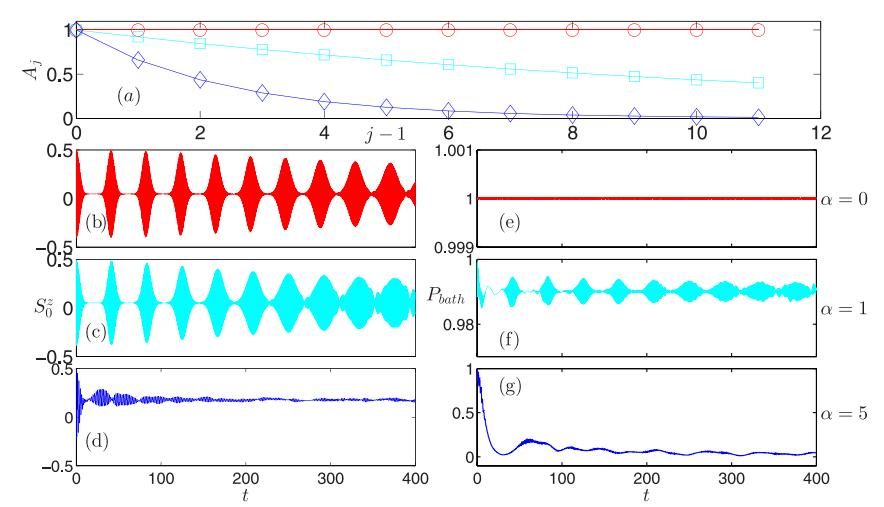

FIG. 4. Effect of inhomogeneity on the CR dynamics. (a) Coupling strengths $A_{j}=A \exp [-\alpha(j-1) / N]$, with $\alpha=0,1,5$, corresponding to homogeneous, intermediate, and strongly inhomogeneous couplings, respectively. (b)-(d) Time evolution of $S_{0}^{z}$ obtained from Eq. (1) using $\Delta_{j}=A_{j}, A=1, N=12, B=1$, and the three chosen values of $\alpha$. (e)-(g) Expectation values of the collective bath projector.

to a breakdown of CR dynamics; see Fig. 4(d). However, we also find that with $\Delta_{j}=A_{j}$ the revivals are remarkably robust to moderate values of the inhomogeneity, $\alpha \sim 1$.

To shed some light on the origin of such behavior, we introduce the collective bath projector $\hat{P}_{\text {bath }}=\sum_{n}|n\rangle\langle n|$ onto the states with a maximal eigenvalue of $\mathbf{J}^{2}$. For a large inhomogeneity factor $\alpha$, the expectation value

$$
P_{\text {bath }}(t)=\left\langle\hat{P}_{\text {bath }}\right\rangle=\sum_{n}|\langle n \mid \psi(t)\rangle|^{2}
$$

quickly decays to small values; see Fig. 4(g). Instead, persistence of $P_{\text {bath }} \sim 0.99$ shown in Fig. 4(f) corresponds to the robust CR dynamics of panel (c). Interestingly, the decay of $P_{\text {bath }}(t)$ with inhomogeneous couplings depends sensitively on the isotropy of the interactions, being much quicker in the Ising case $\left(A_{j}=0\right)$ [58]. We also obtain evidence from the numerical simulations $(N \leqslant 12)$ that the $\mathrm{CR}$ dynamics at given $\alpha \neq 0$ persists for a longer time with a larger number of bath spins. These observations reveal the subtle dependence of CR with respect to the Hamiltonian parameters. In the SM [58] we also explore how CR signatures appear by gradually increasing the degree of bath spin polarization.

In summary, we have obtained the benchmark quantum dynamics of the XXZ central spin problem with homogeneous and inhomogeneous coupling amplitudes. Analytical results of quantum CR, entanglement entropy, and fidelity provide rich insights into quantum dynamic control of entangled states for quantum metrology. The effects of inhomogeneity on the robustness and decay of the CR have been studied as well. Our methods can be directly applied to high central spin problems as well as models of multiple atoms coupled to a cavity in quantum nonlinear optics.

\section{ACKNOWLEDGMENTS}

We thank Henrik Johannesson, Chao-Hong Lee, ShiZhong Zhang, and Li You for helpful discussions. This work is supported by the National Key R\&D Program 
of China No. 2017YFA0304500, the key NSFC Grant No. 11534014, and NSFC Grant No. 11874393. S.C. acknowledges support from the National Key R\&D Program of China No. 2016YFA0301200 and NSFC (Grants No. 11574025, No. 11750110428, and No. 1171101295).
H.-Q.L. and S.C. acknowledge financial support from NSAF U1530401 and computational resources from the Beijing Computational Science Research Center (CSRC). X.-W.G. thanks CSRC, where part of this paper was prepared, for kind hospitality.
[1] M. O. Scully and M. S. Zubairy, Quantum Optics (Cambridge University Press, Cambridge, 1997).

[2] A. Polkovnikov, K. Sengupta, A. Silva, and M. Vengalattore, Rev. Mod. Phys. 83, 863 (2011).

[3] D. Suter and G. A. Álvarez, Rev. Mod. Phys. 88, 041001 (2016).

[4] W. H. Zurek, Nature (London) 317, 505 (1985).

[5] W. Yang, W.-L. Ma, and R.-B. Liu, Rep. Prog. Phys. 80, 016001 (2017)

[6] A. Faribault and D. Schuricht, Phys. Rev. Lett. 110, 040405 (2013).

[7] L. P. Lindoy and D. E. Manolopoulos, Phys. Rev. Lett. 120, 220604 (2018).

[8] W. A. Coish, J. Fischer, and D. Loss, Phys. Rev. B 81, 165315 (2010)

[9] J. H. Eberly, N. B. Narozhny, and J. J. Sanchez-Mondragon, Phys. Rev. Lett. 44, 1323 (1980).

[10] J. Gea-Banacloche, Phys. Rev. Lett. 65, 3385 (1990).

[11] G. Rempe, H. Walther, and N. Klein, Phys. Rev. Lett. 58, 353 (1987).

[12] B. W. Shore and P. Knight, J. Mod. Opt. 40, 1195 (1993).

[13] F. A. A. El-Orany and M. S. Abdalla, J. Phys. A 44, 035302 (2011).

[14] S. Dooley, F. McCrossan, D. Harland, M. J. Everitt, and T. P. Spiller, Phys. Rev. A 87, 052323 (2013).

[15] H. T. Quan, Z. Song, X. F. Liu, P. Zanardi, and C. P. Sun, Phys. Rev. Lett. 96, 140604 (2006).

[16] X. J. Wang, S. Chesi, and W. A. Coish, Phys. Rev. Lett. 109, 237601 (2012).

[17] S. Chesi and W. A. Coish, Phys. Rev. B 91, 245306 (2015).

[18] N. Wu, A. Nanduri, and H. Rabitz, Phys. Rev. A 89, 062105 (2014).

[19] N. Wu, N. Fröhling, X. Xing, J. Hackmann, A. Nanduri, F. B. Anders, and H. Rabitz, Phys. Rev. B 93, 035430 (2016).

[20] N. Wu, Physica A 501, 308 (2018).

[21] A. Blais, R.-S. Huang, A. Wallraff, S. M. Girvin, and R. J. Schoelkopf, Phys. Rev. A 69, 062320 (2004).

[22] C. Neill et al., Nat. Phys. 12, 1037 (2016).

[23] C. Song, K. Xu, W. Liu, C.-P. Yang, S.-B. Zheng, H. Deng, Q. Xie, K. Huang, Q. Guo, L. Zhang, P. Zhang, D. Xu, D. Zheng, X. Zhu, H. Wang, Y.-A. Chen, C.-Y. Lu, S. Han, and J.-W. Pan, Phys. Rev. Lett. 119, 180511 (2017).

[24] K. Xu et al., Phys. Rev. Lett. 120, 050507 (2018).

[25] Z. L. Xiang, S. Ashhab, J. Q. You, and F. Nori, Rev. Mod. Phys. 85, 623 (2013).

[26] M. Saffman, T. G. Walker, and K. Mølmer, Rev. Mod. Phys. 82, 2313 (2010).

[27] D. Barredo, H. Labuhn, S. Ravets, T. Lahaye, A. Browaeys, and C. S. Adams, Phys. Rev. Lett. 114, 113002 (2015).

[28] L. Amico and R. Fazio, J. Phys. A 34, 6425 (2001).
[29] L. Amico, A. Di Lorenzo, and A. Osterloh, Phys. Rev. Lett. 86, 5759 (2001).

[30] L. Amico, A. D. Lorenzo, A. Mastellone, and A. Osterloh, Ann. Phys. 299, 228 (2002).

[31] H.-Q. Zhou, J. Links, R. H. McKenzie, and M. D. Gould, Phys. Rev. B 65, 060502(R) (2002).

[32] W.-L. Yang, Y.-Z. Zhang, and M. D. Gould, Nucl. Phys. B 698, 503 (2004).

[33] J. Dukelsky, S. Pittel, and G. Sierra, Rev. Mod. Phys. 76, 643 (2004).

[34] A. V. Khaetskii, D. Loss, and L. Glazman, Phys. Rev. Lett. 88, 186802 (2002).

[35] A. V. Khaetskii, D. Loss, and L. Glazman, Phys. Rev. B 67, 195329 (2003)

[36] M. Bortz and J. Stolze, Phys. Rev. B 76, 014304 (2007).

[37] M. Bortz, S. Eggert, C. Schneider, R. Stübner, and J. Stolze, Phys. Rev. B 82, 161308(R) (2010).

[38] P. W. Claeys, S. De Baerdemacker, M. Van Raemdonck, and D. Van Neck, J. Phys.: Conf. Ser. 597, 012025 (2015).

[39] P. W. Claeys, S. De Baerdemacker, M. Van Raemdonck, and D. Van Neck, Phys. Rev. B 91, 155102 (2015).

[40] N. M. Bogoliubov and P. P. Kulish, J. Math. Sci. 192, 14 (2013).

[41] J.-B. Yuan, H.-J. Xing, L.-M. Kuang, and S. Yi, Phys. Rev. A 95, 033610 (2017).

[42] T. Kinoshita, T. Wenger, and D. S. Weiss, Nature (London) 440 , 900 (2006).

[43] S. Hofferberth, I. Lesanovsky, B. Fischer, T. Schumm, and J. Schmiedmayer, Nature (London) 449, 324 (2007).

[44] J. P. Ronzheimer, M. Schreiber, S. Braun, S. S. Hodgman, S. Langer, I. P. McCulloch, F. Heidrich-Meisner, I. Bloch, and U. Schneider, Phys. Rev. Lett. 110, 205301 (2013).

[45] P. W. Claeys, S. De Baerdemacker, O. E. Araby, and J.-S. Caux, Phys. Rev. Lett. 121, 080401 (2018).

[46] O. A. Castro-Alvaredo, B. Doyon, and T. Yoshimura, Phys. Rev. X 6, 041065 (2016).

[47] B. Bertini, M. Collura, J. De Nardis, and M. Fagotti, Phys. Rev. Lett. 117, 207201 (2016).

[48] J. Links, SciPost Phys. 3, 007 (2017).

[49] R. W. Richardson and N. Sherman, Nucl. Phys. 52, 221 (1964).

[50] M. Gaudin, J. Phys. 37, 1087 (1976).

[51] M. Gaudin, Bethe Wavefunction (Cambridge University Press, Cambridge, 2014).

[52] G. Kucsko, S. Choi, J. Choi, P. C. Maurer, H. Zhou, R. Landig, H. Sumiya, S. Onoda, J. Isoya, F. Jelezko, E. Demler, N. Y. Yao, and M. D. Lukin, Phys. Rev. Lett. 121, 023601 (2018).

[53] The Zeeman terms of the central spin and the bath spins are in general given by $B s_{0}^{z}+B^{\prime} \sum_{i=1}^{N} S_{i}^{z}=\left(B-B^{\prime}\right) s_{0}^{z}+B^{\prime}\left(s_{0}^{z}+\right.$ $\left.\sum_{i=1}^{N} S_{i}^{z}\right)$. In the Hamiltonian (1) and (2), we dropped the total Zeeman energy term, and thus the effective magnetic field of the central spin was shifted by $\left(B-B^{\prime}\right)$. 
[54] G. Q. Liu, J. Xing, W. L. Ma, P. Wang, C.-H. Li, H. C. Po, Y.-R. Zhang, H. Fan, R.-B. Liu, and X.-Y. Pan, Phys. Rev. Lett. 118, 150504 (2017).

[55] J. A. Jones, S. D. Karlen, J. Fitzsimons, A. Ardavan, S. C. Benjamin, G. Andrew D. Briggs, and J. J. L. Morton, Science 324, 1166 (2009).

[56] W. A. Coish, D. Loss, E. A. Yuzbashyan, and B. L. Altshuler, J. Appl. Phys. 101, 081715 (2007).

[57] A. P. Tonel, J. Links, and A. Foerster, J. Phys. A 38, 1235 (2005).

[58] See Supplemental Material at http://link.aps.org/supplemental/ 10.1103/PhysRevB.99.174308 for a derivation of the exact quantum dynamics of the XXZ central spin problem, and an analysis of the entanglement transfer and dynamical control of the entangled states. The results on the inhomogeneous central spin problem are also included.

[59] L. Childress, M. V. Gurudev Dutt, J. M. Taylor, A. S. Zibrov, F. Jelezko, J. Wrachtrup, P. R. Hemmer, and M. D. Lukin, Science 314, 281 (2006).

[60] H. Bluhm, S. Foletti, I. Neder, M. Rudner, D. Mahalu, V. Umansky, and A. Yacoby, Nat. Phys. 7, 109 (2011).

[61] L. Cywiński, W. M. Witzel, and S. Das Sarma, Phys. Rev. Lett. 102, 057601 (2009).

[62] We shifted the energy of the Hamiltonian (1) by a conserved quantity $h\left(\mathbf{s}_{0}^{z}+\mathbf{a}^{\dagger} \mathbf{a}\right)$.

[63] L. Amico and K. Hikami, Eur. Phys. J. B 43, 387 (2005). 